\title{
Avaliação da adesão de Candida albicans na superfície de resinas acrílicas termopolimerizáveis tratadas com glaze fotopolimerizável e/ou adição de nanopartículas de sílica: um estudo in vitro
}

\author{
- Helidior Teixeira Lopes Departamento de Odontologia, Centro Universitário da Serra Gaúcha (FSG), Caxias do Sul, \\ RS, Brasil • Liliane Alves Departamento de Odontologia, Centro Universitário da Serra Gaúcha (FSG), Caxias do Sul, \\ RS, Brasil • Alexandra Flávia Gazzoni Laboratório de Patologia e Microbiologia Oral, Centro Universitário da Serra \\ Gaúcha (FSG), Caxias do Sul, RS, Brasil
}

RESUMO | Objetivo:Avaliar a adesão de Candida albicans na superfície do poli(metilmetacrilato de metila) após adição de nanopartículas de sílica $0,5 \%$ e/ou selamento com glaze. Materiais e métodos: Foram confeccionados 40 corpos de provas com dimensões de $65 \mathrm{~mm} \times 10 \mathrm{~mm} \times 2,5 \mathrm{~mm}$, subdivididos em 4 grupos: grupo controle $(\mathrm{G} 1)$; grupo sílica (o,5\% de sílica ) (G2), grupo glaze (G3), grupo sílica (o,5\% peso de sílica) + glaze (G4). Os corpos de prova foram imersos separadamente em tubos Falcon que continham uma solução previamente padronizada contendo $10^{7}$ células $/ \mathrm{ml}$ e incubados a $37^{\circ} \mathrm{C}$ em aerobiose por 90 minutos. Após este período, os corpos foram removidos e, posteriormente, analisou-se a adesão fúngica por meio da contagem de Unidades Formadoras de Colônia. Para análise dos dados foi utilizado o teste de ANOVA One-way seguido de comparações múltiplas pelo teste de Tukey $(\mathrm{P} \leq \mathrm{0}, 05)$. Resultados: $\mathrm{O}$ grupo $\mathrm{G} 1$ apresentou diferença estatística significativa para aderência fúngica em comparação aos grupos G2, G3 e G4 (P s o,ooo1). Quando comparados os grupos G2, G3 e G4 entre si, não se observou diferença entre eles. Conclusões: A adição de sílica e/ou glaze sobre a superfície do poli(metilmetacrilato de metila) diminui a adesão da Candida albicans, servindo de estratégia para redução dos índices de lesões bucais ocasionadas pelo uso de próteses odontológicas acrílicas.

DESCRITORES | Candidíase Oral; Resinas Acrílicas; Aderência Celular; Sílica.

ABSTRACT | Adhesion evaluation of Candida albicans on the surface of termopolymerizable acrylic resins treated with photopolymerizable coatings and/or silica nanoparticles addition: an in vitro study • To evaluate how the addition of $0.5 \%$ silica nanoparticles and/or photopolymerizable coatings affect the adhesion of Candida albicans on the surface of acrylic resin PMMA (poly-methyl methacrylate). Materials and methods: A total of 40 specimens were fabricated $(65 \mathrm{~mm} \times 10 \mathrm{~mm} \times 2,5 \mathrm{~mm})$ and randomly divided into four experimental groups according to surface treatment using silica and/or photopolymerizable coating: the control group (G1); silica group (G2) (0.5\% silica); photopolymerizable coating group (G3); and silica group (0.5\% silica) + photopolymerizable coatings (G4). Specimens were separately incubated with a Candida albicans suspension spectrophotometrically standardized with $1 \times 10^{7} \mathrm{CFU} / \mathrm{ml}$ at $37^{\circ} \mathrm{C}$ for 90 minutes. After this period, the specimens were removed and then their fungal adhesion was analyzed by the colonyforming units counting. Data were analyzed by the One-way ANOVA test followed by the Tukey test $(\mathrm{P} \leq 0,05)$. Results: A significant reduction in Candida albicans adhesion was observed in $\mathrm{G}_{2}, \mathrm{G}_{3}$, and $\mathrm{G}_{4}$ when compared to the control group $\mathrm{G} 1$ (P $\leq$ 0.00o1). However, similar fungal adhesion was observed when $\mathrm{G} 2, \mathrm{G} 3$, and $\mathrm{G} 4$ were compared $(\mathrm{P} \leq \mathrm{0.05})$. Conclusions: The addition of silica nanoparticles and/or photopolymerizable coating on the surface of PMMA decreases the adhesion of Candida albicans, which can be used as a strategy to reduce oral lesions caused by acrylic dentures.

DESCRIPTORS | Oral Candidiasis; Acrylic Resins; Cell Adhesion; Silica.

AUTORA CORRESPONDENTE | • Alexandra Flávia Gazzoni Departamento de Odontologia, Centro Universitário da Serra Gaúcha (FSG) - Rua Os Dezoito do Forte, 2366 Caxias do Sul, RS Brasil • 95020-472 E-mail: alexandra.gazzoni@fsg.edu.br

- Received Maio 6, 2019 • ACCEPTED Set. 4, 2019

- Dol http://dx.doi.org/10.11606/issn.2357-8041.clrd.2019.157790 


\section{INTRODUÇÃO}

Em 1930 foi descoberto o poli(metilmetacrilato de metila) (PMMA) a partir do monômero de metil metacrilato. ${ }^{1-3}$ Desde então, as resinas acrílicas termopolimerizáveis são utilizadas para confecção e reembasamento de próteses dentais devido às propriedades da matéria-prima, uma vez que ela apresenta características estéticas particulares, cor aceitável e estabilidade dimensional. ${ }^{4-6}$

Após a confecção de próteses dentárias é necessário acabamento e polimento. Esta conduta tem como objetivos remover os defeitos, bem como eventuais ranhuras na superfície. Assim, o procedimento incrementa três benefícios para a prótese dentária: saúde gengival, função e estética. ${ }^{2}$ Entretanto, mesmo após realização de acabamento minucioso, estudos ${ }^{7,8}$ têm destacado a elevada incidência de estomatites protéticas em pacientes portadores de algum tipo de prótese acrílica odontológica, cujo principal agente causador é a Candida albicans, responsável por uma prevalência que varia entre 15 e $75 \%$ entre estes pacientes.79

À vista disso, os polímeros de metacrilato vêm sofrendo inúmeras alterações para aprimoramento das suas propriedades mecânicas, sempre objetivando a diminuição da formação e/ou evolução do biofilme microbiano. ${ }^{9-13}$ Assim, as nanopartículas são utilizadas como materiais de preenchimento, principalmente em matrizes de resina acrílica termopolimerizáveis. ${ }^{12,14}$ Neste contexto, estudos internacionais s $^{2,3,11,14-16}$ atrelados à engenharia dos materiais se concentram na utilização de nanopartículas de sílica $\left(\mathrm{SiO}_{2}\right)$, uma vez que esta é aplicada em ensaios biológicos em resina acrílica termopolimerizável devido a sua biocompatibilidade, bem como a sua apresentação em nanoesferas. Entretanto, na área microbiológica, a atividade das nanopartículas de carga incorporada à matriz do PMMA está associada a uma ação provocada pelo contato entre os microrganismos e a superfície da resina acrílica.$^{12}$ Logo, quando as nanopartículas entram em contato com a mucosa oral ocorre a inibição de células fúngicas. Tal característica devese à influência na morfologia e na proliferação de leveduras aderidas exercida pela nanopartícula, a qual impede o comportamento fenotípico chave da C. albicans. ${ }^{17}$

Para além disso, áreas de crescimento vegetativo das mais variadas espécies de Candida spp. são também isoladas com muita frequência a partir da superfície interna de próteses totais, fato que está associado à alta taxa de infecções recidivantes. ${ }^{18-19}$ Logo, a recorrência da candidíase relacionada à prótese dentária, após o término da terapia antifúngica, indica que a condição de esterilidade também está ligada à inativação dos microrganismos fúngicos presentes nas próteses. ${ }^{20}$ Neste contexto, o glaze é um verniz fotopolimerizável à base de metilmetacrilato utilizado para selar superfícies de próteses confeccionadas à base de resinas acrílicas termopolimerizáveis ou autopolimerizáveis. Este material sela os poros e elimina eventuais ranhuras pós-acabamento e polimento. ${ }^{21}$ Desta forma, a aplicação do glaze interfere na aderência microbiana, diminuindo-a, bem como proporciona melhores condições de higiene bucal para os usuários de próteses odontológicas. No entanto, a ampla aplicação do verniz não é comumentemente difundida na área odontológica, e a literatura sobre sua interferência na adesão de microrganismos fúngicos como a C. albicans ${ }^{22,23}$ também é escassa.

Tendo em vista a hipótese de que a adição de nanopartícula de $\mathrm{SiO} 2$ associada à aplicação do glaze fotopolimerázel impediria a formação e a evolução do biofilme formado pela $C$. albicans sobre superfície de resina acrílica, este trabalho avalia a adesão de C. albicans após adição de nanosílica a 0,5\% e/ou tratamento com glaze fotopolimerizável sobre superfície de resinas acrílicas termopolimerizáveis, uma vez que não se conhecem estudos que 
relacionam o uso de ambas incorporações associadas ou de modo individualizado na adesão fúngica.

\section{MATERIAIS E MÉTODOS}

\section{Preparo dos corpos de prova}

Foram confeccionados 40 corpos de prova a partir de padrões de cera medindo $70 \mathrm{~mm} \times 30 \mathrm{~mm} \times 2,5 \mathrm{~mm}$, que foram incluídos na base da mufla para micro-ondas (Vipi-STG, Vipi, Pirassununga, SP, Brasil) e em gesso tipo pedra, cuja completa cristalização levou 45 minutos. Depois deste procedimento, fechou-se a contra-mufla que, posteriormente, foi preenchida com gesso especial tipo IV (Durone, Dentsply, Petrópolis, RJ, Brasil). Após 45 minutos, quando o gesso apresentava presa final, foi realizada a eliminação da cera pela técnica do micro-ondas (Panasonic, Manaus, AM, Brasil), inserindo a mufla no micro-ondas em potência baixa por quinze minutos. Subsequentemente, aplicouse uma fina película de isolante (Vipi Film, Vipi, Pirassununga, SP, Brasil) em razão de sua atuação como agente de separação de gesso.

A resina acrílica termopolimerizável Trilux Base (Vipi, Pirassununga, SP, Brasil) foi empregada para confecção dos corpos de prova na proporção preconizada pelo fabricante. Foram inseridos em um recipiente de vidro para mistura $7 \mathrm{ml}$ do monômero e adicionados $14 \mathrm{~g}$ da resina rosa claro com veias. Aguardou-se então até a fase plástica para que os espaços vazios no gesso fossem preenchidos. Posteriormente, realizou-se o fechamento da mufla, que foi estabilizada em uma prensa com carga de uma tonelada.

Após 24 horas, considerado o tempo de descanso do material, realizou-se a polimerização utilizando a mesma técnica, inserindo a mufla no micro-ondas por quinze minutos em potência baixa.

Após a retirada dos corpos de prova, realizaram-se os procedimentos de acabamento e polimento. Tais condutas foram realizadas para ocasionar a regularização e remoção de imperfeições, tais como ondulações e lâminas de excesso de resina acrílica. Para tanto, seguiramse as seguintes etapas: a) fresas de corte fino (Besser, Blumenau, SC, Brasil); b) pontas de pedra montadas de granulação fina (Besser, Blumenau, $\mathrm{SC}$, Brasil); c) secção dos corpos de provas em porções menores com disco diamantado (Besser, Blumenau, SC, Brasil); d) desgaste com fresas de corte fino (Besser, Blumenau, SC, Brasil); e) polimento com tiras de lixas d'água de 180, 320 e 600 granulações. Desta forma, alcançou-se a uniformidade dos corpos de provas, que chegaram a dimensões finais de $65 \mathrm{~mm} \times 10 \mathrm{~mm} \times 2,5 \mathrm{~mm}$, conforme a norma $\mathrm{n}^{\mathrm{o}} 12$ da American Dental Association (ADA). ${ }^{24}$

Os procedimentos de polimento foram realizados em um torno de bancada de uma única velocidade (Protécni, Araraquara, SP, Brasil). Foi adotada a seguinte sequência de polimento para resinas acrílicas termopolimerizáveis: escova de polimento com pedra-pomes umedecida (Kota, Cotia, SP, Brasil); flanela de pano com pedra-pomes umedecida (Kota, Cotia, SP, Brasil); flanela com maior densidade (Kota, Cotia, SP, Brasil) com bastão de polimento (Vipi, Pirassununga, SP, Brasil); flanela de algodão (Kota, Cotia, SP, Brasil) com Branco de Espanha (Kota, Cotia, SP, Brasil) para promover o brilho final.

\section{Adição das nanopartículas de sílica $\left(\mathrm{SiO}_{2}\right)$}

Para a incorporação de nanopartícula de $\mathrm{SiO}_{2}$ (CAB-O-SIL ${ }^{\circledR}$ TS610; Cabot Brasil Ind. e Com., Mauá, SP, Brasil) (pureza de 99,9\%), seguiu-se a metodologia previamente publicada por Cevik e Yildrim-Bicer ${ }^{2}$. Para tanto, as nanopartículas foram incorporadas ao monômero líquido, cujo procedimento de mistura foi realizado de modo manual em recipiente vítreo utilizando uma espátula de aço inoxidável. Posteriormente, o frasco foi agitado por vibração ultrassônica por uma hora com a finalidade de obter uma dispersão mais uniforme. 
Em seguida, tal mistura foi adicionada ao pó acrílico. A relação pó/líquido/ $\mathrm{SiO}_{2}$ foi de 14 g/7 ml/o,07 g.

\section{Método de aplicação do glaze fotopolimerizável}

Para o selamento da superfície dos corpos de prova, foi aplicado o glaze fotopolimerizável (Megaseal, Odonto Mega, Ribeirão Preto, SP, Brasil) como tratamento de superfície. Os corpos de prova pertencentes ao G3 e G4 passaram por aplicação uniforme e delgada em sentido único utilizando um pincel macio. Aguardaram-se vinte segundos para realização de fotoativação durante cinco minutos utilizando um fotopolimerizador com lâmpadas ultravioletas (UVA) (EDG Lux, EDG, São Carlos, SP, Brasil) (Figura 1).

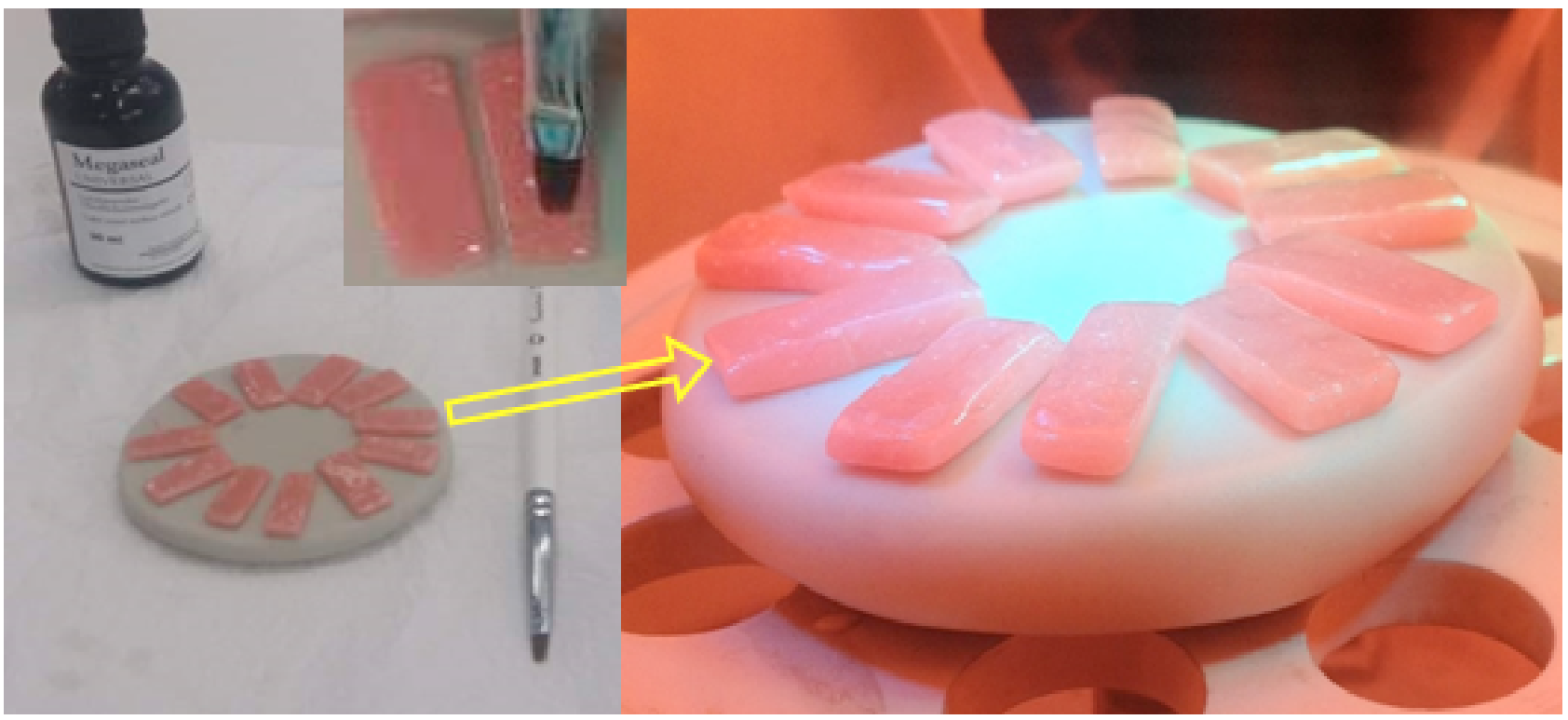

Figura 1 | Aplicação do glaze sobre a superfície dos corpos de prova para a fotopolimerização.

\section{Subdivisão dos corpos de prova}

Os corpos de provas foram subdivididos em quatro grupos, conforme o tratamento de superfície (Tabela 1).

Tabela 1 | Composição dos grupos de estudos

\begin{tabular}{l|l} 
Grupo de estudo & \multicolumn{1}{c}{ Descrição } \\
Grupo 1 (G1) & $\begin{array}{l}10 \text { corpos de prova compostos somente por } \\
\text { PMMA }\end{array}$ \\
\hline Grupo 2 (G2) & $\begin{array}{l}10 \text { corpos de prova compostos por } \\
\text { PMMA + } \mathrm{SiO}_{2}(0,5 \% \text { peso/volume })\end{array}$ \\
\hline Grupo 3 (G3) & $\begin{array}{l}10 \text { corpos de prova compostos por } \\
\text { PMMA + tratamento de superfície com glaze } \\
\text { fotopolimerizável }\end{array}$ \\
\hline Grupo (G4) & $\begin{array}{l}10 \text { corpos de prova compostos } \\
\text { por PMMA + SiO } \\
\text { volume) }(0,5 \% \text { tratamento de superfície com } \\
\text { glaze fotopolimerizável }\end{array}$ \\
\hline
\end{tabular}

PMMA: poli(metacrilato de metila). $\mathrm{SiO}_{2}$ : dióxido de silício.

\section{Aderência de Candida albicans sobre os corpos de prova}

Para analisar a aderência fúngica, foi utilizada uma cepa de C. albicans (ATCC 90028) (Microbiologics ${ }^{\circledR}$, St. Cloud, EUA), reisolada em Ágar Sabouraud Dextrose $4 \%$ adicionado a cloranfenicol (Difco, Le Ponte de Craix, França) e incubada a $37{ }^{\circ} \mathrm{C}$ por 48 horas. Após este período, foram preparadas suspensões fúngicas com concentração de $10^{7}$ leveduras/mililitro, as quais foram padronizadas com uso da escala 0,5 de MacFarland. Para confirmação da concentração de leveduras, utilizou-se contagem em câmara Newbauer (Kasvi, Padova, Itália). Posteriormente, os corpos de prova de todos os grupos do estudo foram imersos em uma solução contendo $1,600 \mu \mathrm{L}$ de caldo infusão cérebro-coração (BHI) (Difco, Le Ponte de Craix, 
França), $200 \mu \mathrm{L}$ de saliva artificial (Bellafarma, Caxias do Sul, RS, Brasil) e $200 \mu \mathrm{L}$ de inóculo fúngico. Em seguida, os corpos de prova foram incubados a $37^{\circ} \mathrm{C}$ por 48 horas, sendo posteriormente, os transferidos para novos tubos Falcon (TPP Techno Plastic Products AG, Transadingen, Suíça) estéreis, nos quais foram adicionados 2,00o $\mu \mathrm{L}$ de água destilada estéril. Após a transferência, os corpos de prova foram submetidos à sonicação (Ultra-Sonic, Unique ${ }^{\circledR}$, Indaiatuba, SP, Brasil) por cinco minutos. Subsequentemente, foi semeada uma alíquota de $100 \mu \mathrm{L}$ do líquido resultante do sonicado em placas de Petri contendo Ágar Sabouraud Dextrose 4\% adicionado a cloranfenicol (Difco, Le Ponte de Craix, França). Em seguida, as placas de Petri foram incubadas a $37^{\circ} \mathrm{C}$ por $24 / 48$ horas. A contagem das unidades formadoras de colônias (UFC/ml) foi realizada de forma manual.

\section{Análise estatística}

Os resultados foram submetidos à análise estatística no programa GraphPad 4.o para Windows (GraphPad Software Inc., La Jolla, CA, EUA). Utilizou-se o teste de Shapiro-Wilk para verificação da distribuição gaussiana dos dados. Posteriormente, para verificar as diferenças na aderência fúngica associada a C. albicans entre os grupos experimentais foi utilizado o teste ANOVA One-way seguido pela análise de comparações múltiplas pelo teste de Tukey.
Os resultados foram considerados estatisticamente significativos quando $\mathrm{P} \leq \mathrm{0}, 05$.

\section{RESULTADOS}

No G1 observou-se uma média de aderência fúngica igual a 3,04 × $10^{10} \mathrm{UFC} / \mathrm{ml}(1,9-6,4 \pm 1,49$; IC 95\%). No G2, o valor médio de adesão da $C$. albicans foi igual a $0,11 \times 10^{10} \mathrm{UFC} / \mathrm{ml}$ (0.05-0,19 \pm 0,05; IC 95\%). No G3, foi observada uma média de microrganismos aderidos de 1,06 × 10 ${ }^{10} \mathrm{UFC} / \mathrm{ml}(0,23-1,9 \pm 0,63$; IC 95\%). No G4, foi observada média de aderência fúngica de $0,18 \times 10^{10} \mathrm{UFC} / \mathrm{ml}(0,04-0,57 \pm 0,17$; IC 95\%). Quando comparados os grupos G1 x G2, G1 x G3 e G1 x G4, observou-se diferença significativa entre eles $(\mathrm{P}<\mathrm{0}, \mathrm{OO0} 1)$. Quando comparados entre si G2, G3 e G4 em relação ao uso da nanopartícula de $\mathrm{SiO}_{2}$ ou glaze fotopolimerizável de modo associado ou individualizado, não foi observada diferença estatisticamente significativa entre os grupos estudados (G2 × G3: -1,941 a 0,03635 (IC 95\%); G2 × G4: -1,061 a 0,9163 (IC 95\%); G3 × G4: -0,1085 a 1,869 (IC 95\%)). A Tabela 2 exemplifica os valores de mínimo, máximo e desvio-padrão encontrados entre os grupos G1, G2, G3 e G4 e mostra a comparação entre os grupos quanto ao grau de adesão fúngica associada à contaminação da superfície dos corpos de provas deste estudo com C. albicans.

Tabela 2 | Valores de mínimo, máximo, média e desvio-padrão encontrados no estudo

\begin{tabular}{c|c} 
Microrganismo & $\begin{array}{c}\text { Grau de adesão da Candida albicans } \\
\text { (ATCC 90028) }\end{array}$ \\
Média (Mínimo-Máximo \pm DP)
\end{tabular}

DP: desvio-padrão.

Letras iguais indicam similaridade estatística. 


\section{DISCUSSÃO}

Os principais estudos microbiológicos demonstram que a evolução da candidíase associada a próteses dentárias tem uma etiologia multifatorial., ${ }^{1,2,6}$ Tal característica deve-se a fatores relacionados ao hospedeiro, tais como: pobre higiene bucal, idade avançada, fumo, mau condicionamento das próteses, assim como a presença de doenças sistêmicas que interferem na ativação da resposta imune. ${ }^{25}$ Desta forma, as lesões provocadas pela C. albicans são comumente encontradas na cavidade oral em regiões como: língua, mucosa jugal, palato mole e/ou palato duro, bem como podem aderir às próteses dentais removíveis ou fixas. ${ }^{18,20}$ Assim, estas lesões apresentam alta taxa de prevalência na cavidade bucal devido a intensa formação de biofilme microbiano, que se torna intensamente profundo e causa irritação das superfícies epidérmicas e mucosas.7,25

No entanto, o desenvolvimento da candidíase associada à prótese dentária não está relacionado somente às características do hospedeiro. As propriedades relacionadas aos materiais utilizados para confecção da prótese também interferem no desenvolvimento e na evolução da candidíase. ${ }^{11-13}$ Nesse sentido, há evidências crescentes na literatura de que uma topografia superficial composta por escala nanométrica é igualmente importante para influenciar o comportamento de crescimento da C. albicans, bem como de células de mamíferos. ${ }^{26}$ Neste contexto, Cevik et al. ${ }^{2}$ e da Silva et al. ${ }^{3}$ avaliaram a resistência, a compressão e a dureza a partir da adição de nanosílica. Eles relataram que tal incorporação tem demonstrado melhora no desempenho mecânico da resina acrílica e concluíram que a nanosílica adicionada a uma concentração de $0,5 \%$ incorpora benefícios diretos na resistência à flexão da resina acrílica apresentando resultados significativos. Logo, optou-se, neste estudo, por adicionar à matriz da resina acrílica uma concentração de $0,5 \%$ de nanosílica. Assim, a partir dos resultados aqui obtidos, comprovou-se que o tratamento com sílica nanoparticulada reduziu significativamente a formação do biofilme fúngico em comparação com corpos de prova do grupo G1 (Grupo Controle). Sob esta ótica, a influência da nanopartícula de sílica na formação do biofilme fúngico está associada à ausência de hifas, que sustentariam o desenvolvimento e a evolução do biofilme. Portanto, essa interferência no comportamento morfológico da levedura resulta em células fúngicas fracamente aderidas que podem ser retiradas da superfície da resina acrílica devido à perda de integridade estrutural fúngica. No entanto, os estudos que focalizam a aderência fúngica sobre resina acrílica após adição de nanosílica a 0,5\% são escassos na literatura, ${ }^{2,11}$ fato que limita outras comparações.

Sobre o uso do glaze fotopolimerizável, dois estudos ${ }^{22,23}$ avaliaram a efetividade de vernizes fotopolimerizáveis experimentais sobre superfície de resina acrílica à base de monômeros hidrofílicos e à base de monômeros zwiteriônicos na redução da adesão de C. albicans. Nesses estudos, os autores observaram que o verniz contendo monômero hidrofílico ou monômero zwiteriônico foram efetivos na redução da formação do biofilme fúngico. Em adição, estudos publicados por Hirota et al. ${ }^{27}$ relatam que o recobrimento superficial com glaze provoca modificações nas resinas acrílicas, que são compatíveis com a diminuição da aderência fúngica. Por sua vez, nossos resultados apoiam os dados publicados por Lanzarin et al. ${ }^{22,23}$ e Hirota et al. ${ }^{27}$, uma vez que também demonstraram diminuição significativa, quando comparado ao grupo controle (G1), na adesão de C. albicans sobre resina acrílica tratada quando o verniz fotopolimerizável foi aplicado à base de metacrilato de metila. Tal redução no número de células aderidas deve-se ao fato de que o verniz fotopolimerizável torna a superfície mais hidrofílica, aspecto que interfere sobremaneira na evolução do biofilme microbiano. 
Quanto aos estudos que consideram as duas incorporações avaliadas nesta pesquisa de forma associada ou de forma individualizada na formação do biofilme provocado por C. albicans, a literatura não apresenta dados correspondentes. No entanto, neste estudo, o uso de ambos os tratamentos de modo associado não apresentou diferença no número de células aderidas em comparação com as incorporações individualizadas. Neste sentido, de acordo com os resultados revelados por Zissis et al., ${ }^{28}$ nenhuma redução da aderência de fungos e bactérias pode ser esperada quando os valores de rugosidade são inferiores a $0,2 \mu \mathrm{m}$, diferença que clinicamente não seria significante para interferir na adesão fúngica. Por isso, foi idealizado neste estudo que tanto a incorporação de nanosílica a $0,5 \%$ quanto o tratamento com verniz fotopolimerizável tornariam a superfície igualmente rugosa e hidrofílica, o que explicaria a não significância dos resultados obtidos para o G2, G3 e G4. Quanto à não significância observada entre $\mathrm{G}_{3}$ e G4, tal resultado está associado à perda parcial do glaze fotopolimerizável quando em imersão, uma vez que a aplicação deste tratamento de superfície originou cobertura uniforme em toda a extensão do corpo de prova.

Por conseguinte, em razão dos resultados obtidos neste estudo e da dificuldade em encontrar relatos na literatura sobre a adesão fúngica aos materiais estudados, fazem-se necessários mais estudos. Para tanto, este estudo pode servir de base para realização de novas pesquisas ou estudos clínicos longitudinais. Assim, nossos resultados podem refletir uma melhora na qualidade de vida de pacientes, reduzindo o índice de lesões bucais ocasionadas pelo uso de próteses acrílicas odontológicas.

\section{CONCLUSÃO}

Conclui-se que há uma diminuição na adesão de Candida albicans sobre a resina acrílica termopolimerizável quando ocorre adição de nanopartícula de sílica a $0,5 \%$, bem como na aplicação de glaze sobre a superfície do PMMA. Assim, a partir dos resultados observados estes materiais tornam-se uma alternativa fácil, barata $\mathrm{e}$ disponível no mercado, podendo ser utilizados na diminuição da aderência fúngica por C. albicans.

\section{REFERÊNCIAS}

1. Pan H, Wang G, Pan J, Ye G, Sun K, Zhang J, et al. Cold plasma-induced surface modification of heat-polymerized acrylic resin and prevention of early adherence of Candida albicans. Dent Mater J. 2015;34(4):529-36. doi:10.4012/dmj.2015-035.

2. Cevik P, Yildirim-Bicer AZ. The effect of silica and prepolymer nanoparticles on the mechanical properties of denture base acrylic resin. J Prosthodont. 2018;27(8):763-70. doi: 10.1111/jopr.12573.

3. da Silva LH, Feitosa SA, Valera MC, de Araújo MA, Tango RN. Effect of the addition of silanated silica on the mechanical properties of microwave heat-cured acrylic resin. Gerodontology. 2012;29(2):e1019-23. doi: 10.1111/j.1741-2358.2011.00604.x.

4. Vanden Abbeele A, de Meel H, Ahariz M, Perraudin JP, Beyer I, Courtois P. Denture contamination by yeasts in the elderly. Gerodontology. 2008;25(4):222-8. doi: 10.1111/j. 1741-2358.2007.00247.x.

5. Koch C, Bürgers R, Hahnel S. Candida albicans adherence and proliferation on the surface of denture base materials. Gerodontology. 2013;30(4):309-13. doi: 10.1111/ger.12056.

6. Mayahara M, Kataoka R, Arimoto T, Tamaki Y, Yamaguchi N, Watanabe $Y$, et al. Effects of surface roughness and dimorphism on the adhesion of Candida albicans to the surface of resins: scanning electron microscope analyses of mode and number of adhesions. J Investig Clin Dent. 2014;5(4):307-12. doi: 10.1111/jicd.12055.

7. Susewind S, Lang R, Hahnel S. Biofilm formation and Candida albicans morphology on the surface of denture base materials. Mycoses. 2015;58(12):719-27. doi: 10.1111/myc.12420.

8. Gendreau L, Loewy ZG. Epidemiology and etiology of denture stomatitis. J Prosthodont. 2011;20(4):251-6o. doi: 10.1111/j. 1532-849X.2011.00698.x.

9. Hamid SK, Al-Dubayan AH, Al-Awami H, Khan SQ, Gad MM. In vitro assessment of the antifungal effects of neem powder added to polymethyl methacrylate denture base material. J Clin Exp Dent. 2019;11(2):e170-e178. doi: 10.4317/jced.55458.

10. Qian K, Pan H, Li Y, Wang G, Zhang J, Pan J. Time-related surface modification of denture base acrylic resin trea- 
ted by atmospheric pressure cold plasma. Dent Mater J. 2016;35(1):97-103. doi: 10.4012/dmj.2015-162.

11. Hong RY, Fu HP, Zhang YJ, Liu L, Wang J, Li HZ, et al. Surface-modified silica nanoparticles for reinforcement of PMMA. J Appl Polym Sci. 2007; 105(4):2176-84. doi: 10.1002/ app.26164.

12. Sivakumar I, Arunachalam KS, Sajjan S, Ramaraju AV, Rao B, Kamaraj B. Incorporation of antimicrobial macromolecules in acrylic denture base resins: a research composition and update. J Prosthodont. 2014;23(4):248-90. doi: 10.1111/ jopr.12105.

13. Stieven E, Conde A, Galafassi D, Elsemann EB, Elsemann RB, Gazzoni AF. Análise da utilização de nanopartículas de prata e/ou plasma de baixa pressão e temperatura para a prevenção de Candida albicans em próteses dentárias. R-BITS. 2018; 8(1):67-7.

14. Qasim M, Singh BR, Nagvi AH, Paik P, Das D. Silver nanoparticles embedded mesoporous $\mathrm{SiO} 2$ nanosphere: an effective anticandidal agent against Candida albicans 077. Nanotechnology. 2015;26(28):285102. doi: 10.1088/0957$4484 / 26 / 28 / 285102$.

15. Lee JH, El-Fiqi A, Jo JK, Kim DA, Kim SC, Jun SK, et al. Development of long-term antimicrobial poly(methyl methacrylate) by incorporating mesoporous silica nanocarriers. Dent Mater. 2016;32(12):1564-74. doi: 10.1016/j.dental.2016.09.001.

16. Yodmongkol S, Chantarachindawong R, Thaweboon S, Thaweboon B, Amornsakchai T, Srikhirin T. The effects of silane-SiO2 nanocomposite films on Candida albicans adhesion and the surface and physical properties of acrylic resin denture base material. J Prosthet Dent. 2014;112(6):1530-8. doi:10.1016/j.prosdent.2014.06.019.

17. Cousins BG, Allison HE, Doherty PJ, Edwards C, Garvey MJ, Martin DS, et al. Effects of a nanoparticulate silica substrate on cell attachment of Candida albicans. J Appl Microbiol. 2007;102(3):757-65. doi: 10.1111/j.1365-2672.2006.03124.x.

18. Monsenego P. Presence of microorganisms on the fitting denture complete surface: study "in vivo”. J Oral Rehabil. 2000:27(8);708-13. doi: 10.1046/j.1365-2842.2000.00564.x.
19. Yano J, Yu A, Fidel PL Jr, Noverr MC. Candida glabrata has no enhancing role in the pathogenesis of Candida-associated denture stomatitis in a rat model. mSphere. 2019;4(2):pii:eoo191-19. doi: 10.1128/mSphere.00191-19.

20. Banting DW, Hill SA. Microwave disinfection of dentures for the treatment of oral candidiasis. Spec Care Dentist. 2001;21(1):4-8.

21. Cağavi F, Akalan N, Celik H, Gür D, Güçiz B. Effect of hydrophilic coating on microorganism colonization in silicone tubing. Acta Neurochir (Wien). 2004;146(6):603-10. doi: 10.1007/s00701-004-0262-z.

22. Lazarin AA, Zamperini CA, Vergani CE, Wady AF, Giampaolo ET, Machado AL. Candida albicans adherence to an acrylic resin modified by experimental photopolymerised coatings: an in vitro study. Gerodontology. 2014;31(1):25-33. doi: 10.111/j.1741-2358.2012.00688.x.

23. Lazarin AA, Machado AL, Zamperini CA, Wady AF, Spolidorio DM, Vergani CE. Effect of experimental photopolymerized coatings on the hydrophobicity of a denture base acrylic resin and on Candida albicans adhesion. Arch Oral Biol. 2013;58(1):1-9. doi: 10.1016/j.archoralbio.212.10.005.

24. Revised American Dental Association specification $n^{0} 12$ for denture base polymers. J Am Dent Assoc. 1975;90(2):39-49. doi: 10.14219/jada.archive.1975.0069.

25. Ramage G, Tomsett K, Wicks BL, López-Ribot JL, Redding SW. Denture stomatitis: a role for Candida biofilms. Oral Surg Oral Med Oral Pathol Oral Radiol Endod. 2004;98(1):53-9. doi: 10.1016/j.tripleo.2003.04.002.

26. Curtis A, Wilkinson C. Nantotechniques and approaches in biotechnology. Trends Biotechnol. 2001;19(3):97-101.

27. Hirota K, Murakami K, Nemoto K, Miyake Y. Coating of a surface with 2-methacryloyloxyethyl phosphorylcholine (MPC) co-polymer significantly reduces retention of human pathogenic microorganisms. FEMS Microbiol Lett. 2005;248(1):37-45. doi: 10.1016/j.femsle.2005.05.019.

28. Zissis AJ, Polyzois GL, Yannikakis SA, Harrison A. Roughness of denture materials: a comparative study. Int J Prosthodont. 2000;13(2):136-40. 